\title{
Shallow-water equations with complete Coriolis force: Group Properties and Similarity Solutions
}

\author{
Andronikos Paliathanasis* \\ Institute of Systems Science, Durban University of Technology \\ PO Box 1334, Durban 4000, Republic of South Africa
}

December 22, 2020

\begin{abstract}
The group properties of the shallow-water equations with the complete Coriolis force is the subject of this study. In particular we apply the Lie theory to classify the system of three nonlinear partial differential equations according to the admitted Lie point symmetries. For each case of the classification problem the one-dimensional optimal system is determined. The results are applied for the derivation of new similarity solutions.
\end{abstract}

Keywords: Lie symmetries; invariants; shallow water; similarity solutions; Coriolis force

\section{Introduction}

Shallow-water equations are a set of hyperbolic nonlinear partial differential equations, known also as the Saint-Venant equations, which describe a thin layer of inviscid fluid with a free surface. There are various applications of the Shallow-water equations in physical science, for some recent results we refer the reader to [1-6] and references therein. In general hyperbolic equations are of special interest for the study of flows. Two-phase flow is of special interest because it is described by a set of hyperbolic equations with non-equilibrium phenomena between different phases. There are various real world applications for these models, hence there are various studies in the literature [7-14].

In this piece of work we are interested in the group properties of the rotating two-dimensional shallow-water equations. The shallow-water equations with the complete Coriolis force term and topography were derived in [15].

In the case of a linear plane the shallow-water equations are

$$
h_{t}+(h u)_{x}+(h v)_{y}=0,
$$

*Email: anpaliat@phys.uoa.gr 


$$
\begin{gathered}
u_{t}+u u_{x}+v u_{x}-\left(2 \Omega_{z}-\Omega_{y} h_{y}\right) v+\left(g h-h^{2} \Omega_{y} u\right)_{x}-\Omega_{y}\left((h u)_{x}+(h v)_{y}\right)=0, \\
v_{t}+u v_{x}+v v_{y}+\left(2 \Omega_{z}-\Omega_{y} h_{y}\right) u+\left(g h-h^{2} \Omega_{y} u\right)_{y}=0
\end{gathered}
$$

where the axes have been selected to be tangent to a sphere at a specific latitude $\phi_{0}$ such that the Coriolis term $\Omega=\left(\Omega_{x}, \Omega_{y}, \Omega_{z}\right)$ has components $\Omega_{x}=0, \Omega_{y}=\Omega \cos \phi_{0}, \Omega_{z}=\Omega \sin \phi_{0}$.

We study the one-parameter point transformations which leave invariant the set of the three equations, (1), (2) and (3). Specifically, the theory of Lie point symmetries is applied in order to determine the admitted Lie point symmetries and the one-dimensional optimal system.

Lie theory is a powerful mathematical treatment for the study of nonlinear differential equations. The existence of a transformation which leaves invariant a set of differential equations indicates the existence of invariant functions, known also as Lie invariants, which can be used to simplify the set of differential equations by reduction of the differential equations. There are various applications of the Lie point symmetries in physical theories [16 23] as also in fluid dynamics and in shallow-water equations. The Lie point symmetries of the non-rotating shallow-water equations were studied in [24, while a similar analysis with specific Coriolis term was performed in [25]. In the latter case it was found that the special Coriolis term can be neglected by the field equations with the use of point transformations. Rotating shallow-water equations with more general fluids were studied in [26] while other studies of shallow-water equations can be found in 24,27,34] and in some recent works in [35] 39. In addition, for the hyperbolic equations which describe the two-phase flow Lie symmetries were applied in [40,41].

Ovsiannikov [42] in 1982 demonstrated the construction of the one-dimensional optimal system for the Lie algebra, using a global matrix for the adjoint transformation. Since then the classification of the one-dimensional optimal system has become a main tool for the study of nonlinear differential equations [43 45]. Such analysis for the shallow-water system has been presented before in [25, 26, 46, 47]. However, in this study we present for the first time the complete symmetry classification for the shallow-water equations with a complete Coriolis term. The structure of the paper is as follows.

In Section 2 we present the basic properties and definitions of Lie's theory. The classification of the Lie point symmetries for the system of our study is presented in Section 3 . We find that in the extreme values of the latitude $\phi_{0}$ the shallow-water equations admit additional Lie point symmetries, while when $\phi_{0}=\frac{\pi}{2}$ we recover previous results of the literature. For all the different cases, we classify the one-dimensional optimal system which is essential to perform reductions and calculate similarity solutions. In Section 4 we apply the Lie invariants to reduce the dependent variables of the shallow-water equations and we present the cases for which similarity solutions can be expressed in terms of closed-form function. Finally in Section 5 we summarize our results and we draw our conclusions.

\section{Preliminaries}

We present the basic definitions and properties for the mathematical tools which we apply in this work. Precisely we give the main definition of point symmetries and of a Lie symmetry vector. Consider now the system $H^{A}$ of partial differential equations with independent variables $y^{i}$ and dependent variables $u^{A}=u^{A}\left(y^{i}\right)$, that is

$$
H^{A}\left(y^{i}, u^{A}, u_{i}^{A}, \ldots\right) \equiv 0,
$$

where $u_{i}^{A}=\frac{\partial u^{A}}{\partial y^{2}}$. 
We define the infinitesimal one-parameter point transformation

$$
\begin{aligned}
\bar{y}^{i} & =y^{i}\left(y^{j}, u^{B} ; \varepsilon\right), \\
\bar{u}^{A} & =u^{A}\left(y^{j}, u^{B} ; \varepsilon\right),
\end{aligned}
$$

defined in the space of variables $\left\{y^{i}, u^{A}\right\}$ in $\varepsilon$ is an infinitesimal parameter. From (5), (6) the infinitesimal transformation is defined

$$
\begin{aligned}
\bar{y}^{i} & =y+\varepsilon \xi^{i}\left(y^{j}, u^{B}\right) \\
\bar{u}^{A} & =u^{A}+\varepsilon \eta^{A}\left(y^{j}, u^{B}\right)
\end{aligned}
$$

with extension in the jet space $\left\{y^{i}, u^{A}, u_{, i}^{A}, \ldots\right\}$ as

$$
\begin{aligned}
\bar{u}_{i}^{A}= & u_{i}^{A}+\varepsilon \eta_{i}^{A[1]} \\
& \ldots \\
\bar{u}_{i_{1} i_{2} \ldots i_{n}}^{A}= & u_{i_{1} i_{2} \ldots i_{n}}^{A}+\varepsilon D_{i} \eta^{A}-u_{i_{1} i_{2} \ldots i_{n-1}}^{A} D_{i}\left(\xi^{j}\right),
\end{aligned}
$$

where

$$
\eta_{i}^{A[1]}=D_{i} \eta^{A}-u_{i_{1} i_{2} \ldots i_{n-1}} D_{i}\left(\xi^{j}\right)
$$

and

$$
\eta^{A[n]}=D_{i} \eta^{A[n-1]}-u_{i_{1} i_{2} \ldots i_{n-1}} D_{i}\left(\xi^{j}\right) .
$$

Under the action of the one-parameter point transformation (5), (6) the system of differential equations becomes $\bar{H}^{A}\left(\bar{y}^{i}, \bar{u}^{A}, \bar{u}_{i}^{A}, \ldots, \varepsilon\right)$. We say that the system $H^{A}$ remains invariant under the action of the oneparameter point transformation (5), (6) if and only if $\bar{H}^{A}\left(\bar{y}^{i}, \bar{u}^{A}, \bar{u}_{i}^{A}, \ldots, \varepsilon\right) \equiv 0$, or equivalently [48 50]

$$
\lim _{\varepsilon \rightarrow 0} \frac{\bar{H}^{A}\left(\bar{y}^{i}, \bar{u}^{A}, \ldots ; \varepsilon\right)-H^{A}\left(y^{i}, u^{A}, \ldots\right)}{\varepsilon}=0
$$

that is,

$$
\mathcal{L}_{X}\left(H^{A}\right)=0,
$$

where $\mathcal{L}$ denotes the Lie derivative with respect the generator $X$ of the infinitesimal transformation (5), (6), that is,

$$
X=\xi^{i}\left(y^{j}, u^{B}\right) \partial_{i}+\eta^{A}\left(y^{j}, u^{B}\right) \partial_{A}
$$

The latter vector field is set in the jet space, $\left\{y^{i}, u^{A}, u_{, i}^{A}, \ldots\right\}$,

$$
X^{[n]}=X+\eta^{[1]} \partial_{u_{i}^{A}}+\ldots+\eta^{[n]} \partial_{u_{i_{i} i_{j} \ldots i_{n}}^{A}} .
$$

When (12) is true for a specific vector field $X$, then the vector field $X$ is a Lie symmetry for the system of partial differential equations $H^{A}$.

The admitted symmetry vectors of a given set of differential equations constitute a closed-group known as a Lie group. A Lie group defines the main properties for the set of the differential equations. The main application of the Lie symmetries is the determination of solutions known as similarity solutions and follow from the application of the Lie invariants in the differential equations. However, in order to classify all the possible similarity transformations and solutions the one-dimensional optimal system should be calculated [48]. 
Table 1: Commutator table for the Lie point symmetries of the Shallow-Water system

\begin{tabular}{cccc}
\hline \hline$\left[X_{I}, X_{J}\right]$ & $\mathbf{X}_{1}$ & $\mathbf{X}_{2}$ & $\mathbf{X}_{3}$ \\
\hline $\mathbf{X}_{1}$ & 0 & 0 & 0 \\
$\mathbf{X}_{2}$ & 0 & 0 & 0 \\
$\mathbf{X}_{3}$ & 0 & 0 & 0 \\
\hline \hline
\end{tabular}

Table 2: Adjoint representation for the Lie point symmetries of the Shallow-Water System

\begin{tabular}{cccc}
\hline \hline $\operatorname{Ad}\left(e^{\left(\varepsilon \mathbf{X}_{i}\right)}\right) \mathbf{X}_{j}$ & $\mathbf{X}_{1}$ & $\mathbf{X}_{2}$ & $\mathbf{X}_{3}$ \\
\hline $\mathbf{X}_{1}$ & $X_{1}$ & $X_{2}$ & $X_{3}$ \\
$\mathbf{X}_{2}$ & $X_{1}$ & $X_{2}$ & $X_{3}$ \\
$\mathbf{X}_{3}$ & $X_{1}$ & $X_{2}$ & $X_{3}$ \\
\hline \hline
\end{tabular}

\section{$3 \quad$ Lie symmetry analysis}

We apply the theory for the system of the three-dimensional partial differential equations, (10), (2) and (3). It follows that the admitted Lie point symmetries for arbitrary latitude $\phi_{0}$ are

$$
X_{1}=\partial_{t}, X_{2}=\partial_{x}, X_{3}=\partial_{y}
$$

In Table 1 we present the commutators for the three-dimensional Lie algebra, $\left\{X_{1}, X_{2}, X_{3}\right\}$. The adjoint representation of the latter Lie algebra is presented in Table 2. From Table 1 we infer that the admitted Lie algebra is the $3 A_{1}$ according to the classification scheme of Patera et al. [51,52.

From tables 1 and 2 we conclude that the one-dimensional optimal system [48] of the four-dimensional Lie algebra consists of the vector fields

$$
\begin{gathered}
\left\{X_{1}\right\},\left\{X_{2}\right\},\left\{X_{3}\right\},\left\{X_{1}+\alpha X_{2}\right\}, \\
\left\{X_{1}+\alpha X_{3}\right\},\left\{X_{2}+\alpha X_{3}\right\},\left\{X_{1}+\alpha X_{2}+\beta X_{3}\right\} .
\end{gathered}
$$

For special values of the parameters $\Omega_{z}$ or $\Omega_{y}$ the resulting Shallow-water system is invariant under a different Lie algebra. We find that the two limits are for the latitudes (i) $\phi_{0}=0$ and (ii) $\phi_{0}=\frac{\pi}{2}$, which correspond to values (i) $\Omega_{y}=\Omega, \Omega_{z}=0$, and (ii) $\Omega_{y}=0, \Omega_{z}=\Omega$.

\subsection{Lie symmetries at the equator}

For latitude $\phi_{0}=0$, that is, for the equator, the resulting Shallow-water equations, (11), (2) and (3), are written as

$$
\begin{gathered}
h_{t}+(h u)_{x}+(h v)_{y}=0, \\
u_{t}+u u_{x}+v u_{x}+\left(\Omega_{y} h_{y}\right) v+\left(g h-h^{2} \Omega_{y} u\right)_{x}-\Omega_{y}\left((h u)_{x}+(h v)_{y}\right)=0, \\
v_{t}+u v_{x}+v v_{y}-\left(\Omega_{y} h_{y}\right) u+\left(g h-h^{2} \Omega_{y} u\right)_{y}=0
\end{gathered}
$$


Table 3: Commutator table for the Lie point symmetries of the Shallow-Water System for $\phi_{0}=0$

\begin{tabular}{cccccc}
\hline \hline$\left[\mathbf{Y}_{I}, \mathbf{Y}_{J}\right]$ & $\mathbf{Y}_{1}$ & $\mathbf{Y}_{2}$ & $\mathbf{Y}_{3}$ & $\mathbf{Y}_{4}$ & $\mathbf{Y}_{5}$ \\
\hline $\mathbf{Y}_{1}$ & 0 & 0 & 0 & $Y_{1}$ & $Y_{3}$ \\
$\mathbf{Y}_{2}$ & 0 & 0 & 0 & $Y_{2}$ & 0 \\
$\mathbf{Y}_{3}$ & 0 & 0 & 0 & $Y_{3}$ & 0 \\
$\mathbf{Y}_{4}$ & $-Y_{1}$ & $-Y_{2}$ & $-Y_{3}$ & 0 & 0 \\
$\mathbf{Y}_{5}$ & $-Y_{3}$ & 0 & 0 & 0 & 0 \\
\hline \hline
\end{tabular}

Table 4: Adjoint representation for the Lie point symmetries of the Shallow-water System for $\phi_{0}=0$

\begin{tabular}{cccccc}
\hline \hline $\operatorname{Ad}\left(e^{\left(\varepsilon \mathbf{Y}_{i}\right)}\right) \mathbf{Y}_{j}$ & $\mathbf{Y}_{1}$ & $\mathbf{Y}_{2}$ & $\mathbf{Y}_{3}$ & $\mathbf{Y}_{4}$ & $\mathbf{Y}_{5}$ \\
\hline $\mathbf{Y}_{1}$ & $Y_{1}$ & $Y_{2}$ & $Y_{3}$ & $Y_{4}-\varepsilon X_{1}$ & $Y_{5}-\varepsilon X_{3}$ \\
$\mathbf{Y}_{2}$ & $Y_{1}$ & $Y_{2}$ & $Y_{3}$ & $Y_{4}-\varepsilon X_{2}$ & $Y_{5}$ \\
$\mathbf{Y}_{3}$ & $Y_{1}$ & $Y_{2}$ & $Y_{3}$ & $Y_{4}-\varepsilon X_{3}$ & $Y_{5}$ \\
$\mathbf{Y}_{4}$ & $e^{\varepsilon} Y_{1}$ & $e^{\varepsilon} Y_{2}$ & $e^{\varepsilon} Y_{3}$ & $Y_{4}$ & $Y_{5}$ \\
$\mathbf{Y}_{5}$ & $Y_{1}+\varepsilon X_{3}$ & $Y_{2}$ & $Y_{3}$ & $Y_{4}$ & $Y_{5}$ \\
\hline \hline
\end{tabular}

and admit the following Lie point symmetries

$$
Y_{1}=\partial_{t}, Y_{2}=\partial_{x}, Y_{3}=\partial_{y}, Y_{4}=t \partial_{t}+x \partial_{x}+y \partial_{y}, Y_{5}=t \partial_{y}+\partial_{V}
$$

with commutators and adjoint representation as given in Tables 3 and 4 . The Lie symmetries form the $\left\{3 A_{1} \otimes_{s} 2 A_{1}\right\}$ Lie algebra.

With the use of the adjoint representation from Table 4 we can determine the one-dimensional optimal system, that is,

$$
\begin{gathered}
\left\{Y_{1}\right\},\left\{Y_{2}\right\},\left\{Y_{3}\right\},\left\{Y_{4}\right\},\left\{Y_{5}\right\}, \\
\left\{Y_{1}+a_{2} Y_{2}\right\},\left\{Y_{1}+a_{3} Y_{3}\right\},\left\{Y_{1}+a_{4} Y_{4}\right\}, \\
\left\{Y_{1}+a_{5} Y_{5}\right\},\left\{Y_{2}+a_{3} Y_{3}\right\},\left\{Y_{1}+a_{5} Y_{5}\right\}, \\
\left\{Y_{1}+a_{2} Y_{2}+a_{3} Y_{3}\right\},\left\{a_{1} Y_{1}+a_{4} Y_{4}+a_{5} Y_{5}\right\} .
\end{gathered}
$$

\subsection{Lie symmetries at the pole}

For latitudes near to the pole, i.e. $\phi=\frac{\pi}{2}$, the Shallow-water equations (11), (2) and (3) can be written in the simple form

$$
\begin{gathered}
h_{t}+(h u)_{x}+(h v)_{y}=0, \\
u_{t}+u u_{x}+v u_{x}+g h_{x}-2 \Omega_{z} v=0, \\
v_{t}+u v_{x}+v v_{y}+g h_{y}+2 \Omega_{z} u=0 .
\end{gathered}
$$

The group properties of the latter system were studied before in [25], where in comparison with [25] $f=2 \Omega_{z}$. 
Equations (17), (18) and (19) are invariant under the action of a nine-dimensional Lie algebra with elements

$$
\begin{gathered}
Z_{1}=\partial_{t}, Z_{2}=\partial_{x}, Z_{3}=\partial_{y}, \\
Z_{4}=x \partial_{x}+y \partial_{y}+u \partial_{u}+v \partial_{v}+2 h \partial_{h} \\
Z_{5}=y \partial_{x}-x \partial_{y}+v \partial_{u}-u \partial_{v}, \\
Z_{6}=\sin (2 \Omega t) \partial_{x}+\cos (2 \Omega t) \partial_{y}+2 \Omega\left(\cos (2 \Omega t) \partial_{u}-\sin (2 \Omega t) \partial_{v}\right), \\
Z_{7}=-\cos (2 \Omega t) \partial_{x}+\sin (2 \Omega t) \partial_{y}+2 \Omega\left(\sin (2 \Omega t) \partial_{u}+\cos (2 \Omega t) \partial_{v}\right), \\
Z_{8}=\quad \sin (2 \Omega t) \partial_{t}+\Omega(x \cos (2 \Omega t)+y \sin (2 \Omega t)) \partial_{x}-\Omega(x \sin (2 \Omega t)-y \cos (2 \Omega t)) \partial_{y}+ \\
-\Omega(u \cos (2 \Omega t)-v \sin (2 \Omega t)-2 \Omega(y \cos (2 \Omega t)-x \sin (2 \Omega t))) \partial_{u}+ \\
-\Omega(u \sin (2 \Omega t)+u \cos (2 \Omega t)+2 \Omega(y \sin (2 \Omega t)+x \cos (2 \Omega t))) \partial_{v}-2 \Omega h \cos (2 \Omega t) \partial_{h}, \\
Z_{9}=\quad \cos (2 \Omega t) \partial_{t}+\Omega(y \cos (2 \Omega t)-x \sin (2 \Omega t)) \partial_{x}-\Omega(x \cos (2 \Omega t)+y \sin (2 \Omega t)) \partial_{y}+ \\
+\Omega(u \sin (2 \Omega t)+v \cos (2 \Omega t)-2 \Omega(x \cos (2 \Omega t)+y \sin (2 \Omega t))) \partial_{u}+ \\
+\Omega(-u \cos (2 \Omega t)+v \sin (2 \Omega t)+\Omega(x \sin (2 \Omega t)-y \cos (2 \Omega t))) \partial_{v}+2 \Omega \sin (2 \Omega t) \partial_{h} .
\end{gathered}
$$

The commutators are presented in Table 5

The symmetry vectors form a nine-dimensional Lie algebra. Furthermore, the adjoint representation of the nine-dimensional Lie algebra is given in Tables 6 and 7

The shallow-water equations without a Coriolis term admit also nine Lie point symmetries with the same commutators. Hence, the same Lie algebra with that of the Coriolis term at the pole but in a different representation. That common property has been observed before in [25. The coordinate transformation which relates the two different representations of the Lie algebra can be use to remove the Coriolis term from the system (17), (18) and (19).

We do not continue with the determination of the one-dimensional optimal system for the system (17), (18) and (19), because it can be find in [25]. 
Table 5: Commutator table for the Lie point symmetries of the Shallow-water system for $\phi_{0}=\pi / 2$

\begin{tabular}{cccccccccc}
\hline \hline$\left[\mathbf{Z}_{I}, \mathbf{Z}_{J}\right]$ & $\mathbf{Z}_{1}$ & $\mathbf{Z}_{2}$ & $\mathbf{Z}_{3}$ & $\mathbf{Z}_{4}$ & $\mathbf{Z}_{5}$ & $\mathbf{Z}_{6}$ & $\mathbf{Z}_{7}$ & $\mathbf{Z}_{8}$ & $-2 \Omega Z_{8}$ \\
\hline $\mathbf{Z}_{1}$ & 0 & 0 & 0 & 0 & 0 & $-2 \Omega Z_{7}$ & $2 \Omega Z_{8}$ & $-2 \Omega Z_{9}$ \\
$\mathbf{Z}_{2}$ & 0 & 0 & 0 & $Z_{2}$ & $-Z_{3}$ & 0 & 0 & $-2 \Omega Z_{7}$ \\
$\mathbf{Z}_{3}$ & 0 & 0 & 0 & $Z_{3}$ & $Z_{2}$ & 0 & 0 & 0 \\
$\mathbf{Z}_{4}$ & 0 & $-Z_{2}$ & $-Z_{3}$ & 0 & 0 & $-Z_{6}$ & $-Z_{7}$ & 0 & $-2 \Omega Z_{6}$ \\
$\mathbf{Z}_{5}$ & 0 & $Z_{3}$ & $-Z_{2}$ & 0 & 0 & $(1-2 \Omega) Z_{7}$ & $(1-2 \Omega) Z_{6}$ & $-2 \Omega Z_{8}$ \\
$\mathbf{Z}_{6}$ & $2 \Omega Z_{7}$ & 0 & 0 & $Z_{6}$ & $-(1-2 \Omega) Z_{7}$ & 0 & 0 & $2 \Omega Z_{3}$ \\
$\mathbf{Z}_{7}$ & $-2 \Omega Z_{8}$ & 0 & 0 & $Z_{7}$ & $-(1-2 \Omega) Z_{6}$ & 0 & 0 & $-2 \Omega Z_{2}$ \\
$\mathbf{Z}_{8}$ & $-2 \Omega Z_{9}$ & $2 \Omega Z_{7}$ & $2 \Omega Z_{6}$ & 0 & $2 \Omega Z_{8}$ & $-2 \Omega Z_{3}$ & $2 \Omega Z_{2}$ & $-2 \Omega Z_{3}$ \\
$\mathbf{Z}_{9}$ & $2 \Omega Z_{8}$ & $2 \Omega Z_{6}$ & $2 \Omega Z_{7}$ & 0 & $-2 \Omega Z_{8}$ & $-2 \Omega Z_{2}$ & $2 \Omega Z_{3}$ & $-2 \Omega\left(Z_{1}+2 \Omega Z_{5}\right)$ \\
\hline \hline
\end{tabular}


Table 6: Adjoint representation for the Lie point symmetries of the Shallow-water system for $\phi_{0}=\pi / 2, \operatorname{sh}$ indicates $\sinh$ while $c h$ means $\cosh (1 / 2)$

\begin{tabular}{cccccc}
\hline \hline$A d\left(e^{\left(\varepsilon \mathbf{Z}_{i}\right)}\right) \mathbf{Z}_{j}$ & $\mathbf{Z}_{1}$ & $\mathbf{Z}_{2}$ & $\mathbf{Z}_{3}$ & $\mathbf{Z}_{4}$ & $Z_{4}$ \\
\hline $\mathbf{Z}_{1}$ & $Z_{1}$ & $Z_{2}$ & $Z_{3}$ & $Z_{5}$ \\
$\mathbf{Z}_{2}$ & $Z_{1}$ & $Z_{2}$ & $Z_{3}$ & $Z_{4}-\varepsilon Z_{2}$ & $Z_{5}-\varepsilon Z_{3}$ \\
$\mathbf{Z}_{3}$ & $Z_{1}$ & $Z_{2}$ & $Z_{3}$ & $Z_{4}-\varepsilon Z_{3}$ \\
$\mathbf{Z}_{4}$ & $Z_{1}$ & $e^{\varepsilon} Z_{2}$ & $Z^{\varepsilon} Z_{3}$ & $Z_{5}$ \\
$\mathbf{Z}_{5}$ & $Z_{1}$ & $\cos (\varepsilon) Z_{2}+\sin (\varepsilon) Z_{3}$ & $-\sin (\varepsilon) Z_{2}+\cos (\varepsilon) Z_{3}$ & $Z_{4}$ & $Z_{5}$ \\
$\mathbf{Z}_{6}$ & $Z_{1}-2 \Omega \varepsilon Z_{7}$ & $Z_{2}$ & $Z_{3}$ & $Z_{4}-\varepsilon Z_{6}$ & $Z_{5}$ \\
$\mathbf{Z}_{7}$ & $Z_{1}+2 \Omega \varepsilon Z_{6}$ & $Z_{2}$ & $Z_{3}$ & $Z_{4}-\varepsilon Z_{7}$ \\
$\mathbf{Z}_{8}$ & $c h^{2}(2 \Omega \varepsilon) Z_{1}-s h^{2}(2 \Omega \varepsilon) Z_{5}+\frac{1}{2} \operatorname{sh}(4 \Omega \varepsilon) Z_{9}$ & $c h(2 \Omega \varepsilon) Z_{2}+\operatorname{sh}(2 \Omega \varepsilon) Z_{7}$ & $\operatorname{ch}(2 \Omega \varepsilon) Z_{3}+\operatorname{sh}(2 \Omega \varepsilon) Z_{6}$ & $Z_{4}$ & $-\frac{s h^{2}(2 \Omega \varepsilon)}{2 \Omega} Z_{1}+c h^{2}(2 \Omega \varepsilon) Z_{5}-\frac{s h(4 \Omega \varepsilon)}{42} Z_{9}$ \\
$\mathbf{Z}_{9}$ & $c h^{22}(2 \Omega \varepsilon) Z_{1}-s h^{2}(2 \Omega \varepsilon) Z_{5}+\frac{1}{2} \operatorname{sh}(4 \Omega \varepsilon) Z_{8}$ & $c h(2 \Omega \varepsilon) Z_{2}+\operatorname{sh}(2 \Omega \varepsilon) Z_{6}$ & $\operatorname{ch}(2 \Omega \varepsilon) Z_{3}+\operatorname{sh}(2 \Omega \varepsilon) Z_{7}$ & $Z_{4}$ & $-\frac{s h^{2}(2 \Omega \varepsilon)}{2 \Omega} Z_{1}+c h^{2}(2 \Omega \varepsilon) Z_{5}+\frac{s h(4 \Omega \varepsilon)}{4 \Omega} Z_{8}$ \\
\hline \hline
\end{tabular}


Table 7: Adjoint representation for the Lie point symmetries of the Shallow-water system for $\phi_{0}=\pi / 2$, sh indicates $\sinh$ while $\operatorname{ch}$ means $\cosh (2 / 2)$

\begin{tabular}{|c|c|c|c|c|}
\hline$A d\left(e^{\left(\varepsilon \mathbf{Z}_{i}\right)}\right) \mathbf{Z}_{j}$ & $\mathbf{Z}_{6}$ & $\mathbf{Z}_{7}$ & $\mathbf{Z}_{8}$ & $\mathbf{Z}_{9}$ \\
\hline $\mathbf{Z}_{1}$ & $\cos (2 \Omega \varepsilon) Z_{6}+\sin (2 \Omega \varepsilon) Z_{7}$ & $-\sin (2 \Omega \varepsilon) Z_{6}+\cos (2 \Omega \varepsilon) Z_{7}$ & $\cos (2 \Omega \varepsilon) Z_{8}-\sin (2 \Omega \varepsilon) Z_{9}$ & $\sin (2 \Omega \varepsilon) Z_{8}+\cos (2 \Omega \varepsilon) Z_{9}$ \\
\hline $\mathbf{Z}_{2}$ & $Z_{6}$ & $Z_{7}$ & $Z_{8}+2 \Omega \varepsilon Z_{7}$ & $Z_{9}+2 \Omega \varepsilon Z_{6}$ \\
\hline $\mathbf{Z}_{3}$ & $Z_{6}$ & $Z_{7}$ & $Z_{8}-2 \Omega \varepsilon Z_{6}$ & $Z_{9}+2 \Omega \varepsilon Z_{7}$ \\
\hline $\mathbf{Z}_{4}$ & $e^{\varepsilon} Z_{6}$ & $e^{\varepsilon} Z_{7}$ & $Z_{8}$ & $Z_{9}$ \\
\hline $\mathbf{Z}_{5}$ & $Z_{6}$ & $Z_{7}$ & $\cos (\varepsilon) Z_{8}+\sin (\varepsilon) Z_{9}$ & $-\sin (\varepsilon) Z_{8}+\cos (\varepsilon) Z_{9}$ \\
\hline $\mathbf{Z}_{6}$ & $Z_{6}$ & $Z_{7}$ & $Z_{8}-2 \Omega \varepsilon Z_{3}$ & $Z_{9}+2 \Omega \varepsilon Z_{2}$ \\
\hline $\mathbf{Z}_{7}$ & $Z_{6}$ & $Z_{7}$ & $Z_{8}+2 \Omega \varepsilon Z_{2}$ & $Z_{9}+2 \Omega \varepsilon Z_{3}$ \\
\hline $\mathbf{Z}_{8}$ & $\operatorname{sh}(2 \Omega \varepsilon) Z_{3}+\operatorname{ch}(2 \Omega \varepsilon) Z_{6}$ & $\operatorname{ch}(2 \Omega \varepsilon) Z_{7}-\operatorname{sh}(2 \Omega \varepsilon) Z_{2}$ & $Z_{8}$ & $\operatorname{ch}(4 \Omega \varepsilon) Z_{9}+\operatorname{sh}(4 \Omega \varepsilon)\left(Z_{1}-2 \Omega Z_{5}\right)$ \\
\hline $\mathbf{Z}_{9}$ & $-\operatorname{sh}(2 \Omega \varepsilon) Z_{2}+\operatorname{ch}(2 \Omega \varepsilon) Z_{6}$ & $\operatorname{ch}(2 \Omega \varepsilon) Z_{7}-\operatorname{sh}(2 \Omega \varepsilon) Z_{3}$ & $\operatorname{ch}(4 \Omega \varepsilon) Z_{8}+\operatorname{sh}(4 \Omega \varepsilon)\left(Z_{1}-2 \Omega Z_{5}\right)$ & $Z_{9}$ \\
\hline
\end{tabular}




\section{Similarity solutions}

In this Section we apply the Lie point symmetries to determine similarity solutions for the Shallow-water system, (11), (2) and (3). For arbitrary latitude the admitted Lie point symmetries provide only travelling-wave solutions. Therefore we focus on the case with $\phi_{0}=0$, where there are additional Lie point symmetries which can provide alternate reductions.

In order to determine exact similarity solutions for the original system we need the application of at least two Lie point symmetries. Thus in the following lines we continue with the investigation of exact similarity solutions by using the Lie invariants of the following set of Lie point symmetries $\left\{X_{1}+X_{2}+X_{3}, X_{2}-X_{3}\right\}$, and in the limit at the equator we study similarity solutions from the Lie symmetries $\left\{Y_{2}, Y_{5}\right\},\left\{Y_{2}, Y_{5}\right\},\left\{Y_{4}, Y_{5}\right\}$. The reduced equations are ordinary differential equations and lead to closed-form solutions.

\subsection{Reduction with $\left\{X_{1}+X_{2}+X_{3}, X_{2}-X_{3}\right\}$}

Consider the similarity transformation provided by the set of the symmetry vectors $\left\{X_{1}+X_{2}+X_{3}, X_{2}-X_{3}\right\}$,

$$
h(t, x, y)=H(w), u(t, x, y)=U(w), v(t, x, y)=V(w) \text { with } w=(x+y)-2 t,
$$

while the reduced system is

$$
\begin{gathered}
\frac{G(w)}{2 \Omega_{z}} \frac{H_{w}}{H}=V^{2}-2 V-U^{2}+2 U+\Omega_{y} H^{2} U+\Omega_{y} H^{2} V \\
-\frac{G(w)}{2 \Omega_{z}} U_{w}=2 H U \Omega_{y}(H V+H U+1)-V H g-U H g+U^{2} V+2 U V^{2}-4 U V+4 V+V^{3}-4 V^{2}, \\
-\frac{G(w)}{2 \Omega_{z}} V_{w}=\begin{array}{r}
V H g+U H g-2 U^{2} V-U V^{2}+4 U V-4 U+4 U^{2}-U^{3}+ \\
-H \Omega_{y}\left(U^{2} H+2 U(1+H)+V H(2-V)\right)
\end{array}
\end{gathered}
$$

where

$$
\begin{aligned}
G(w)= & 6 U^{2}+6 V^{2}-12 V-4 H g+12 U V+8-3 U^{2} V-3 U V^{2} \\
& +2 U H g-V^{3}-U^{3}+2 V H g-12 U+ \\
& -H \Omega_{y}(U+V-2)\left(U+3 H U+H\left(2-V-H \Omega_{z}\right)+2-V\right) .
\end{aligned}
$$

In Fig. 1 we present the numerical solution of the travelling-wave solution as it is given by the dynamical system (21), (22) and (22). The numerical solution is for $\phi_{0}=\frac{\pi}{4}$ and $\Omega_{z}=\Omega_{y}$.

\subsection{Reduction with $\left\{Y_{2}, Y_{5}\right\}$}

The application of the Lie point symmetries $\left\{Y_{2}, Y_{5}\right\}$ lead to the invariants

$$
h(t, x, y)=H(t), u(t, x, y)=U(t), v(t, x, y)=\frac{y}{t}+V(t),
$$

where the unknown functions, $H(t), U(t), V(t)$, satisfy the following system

$$
t H_{t}+H=0, t U_{t}-\Omega H=0, t V_{t}+V=0,
$$

with exact solution

$$
H(t)=\frac{H_{0}}{t}, U(t)=-\frac{H_{0} \Omega}{t}+U_{0}, V(t)=\frac{V_{0}}{t} .
$$



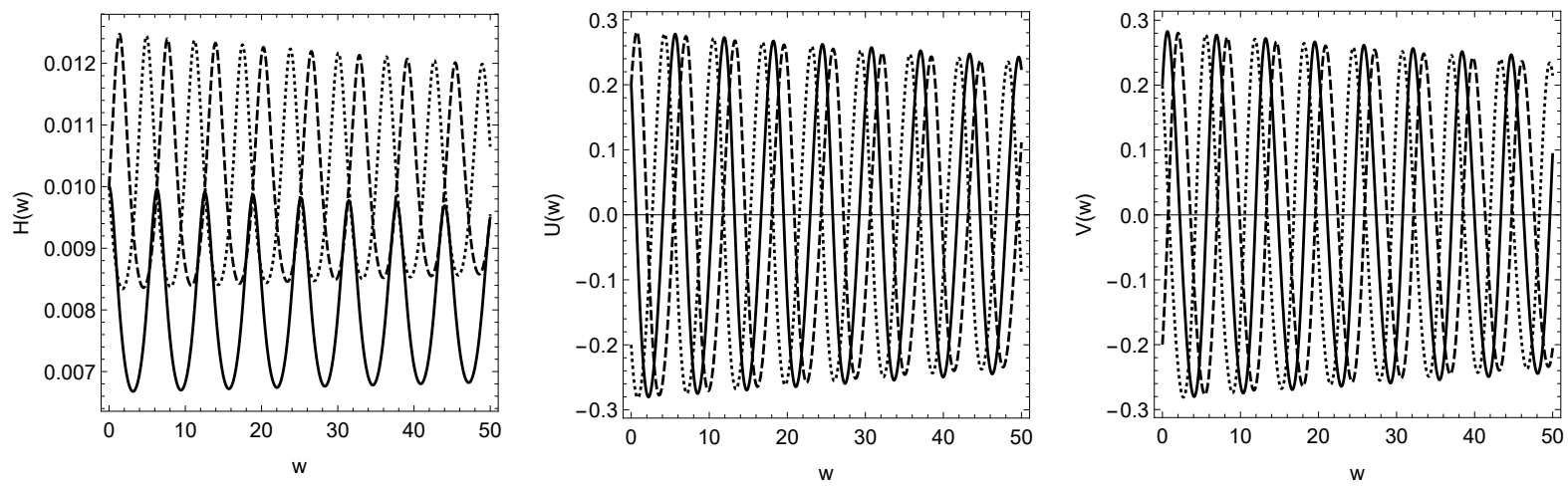

Figure 1: Qualitative evolution of $H(w), U(w), V(w)$ as it is given from numerical simulation of the dynamical system (21), (22) and (22). The plots are for $\Omega_{z}=\Omega_{y}=1$ and $g=10$ and for different initial conditions as they are presented at the plots.
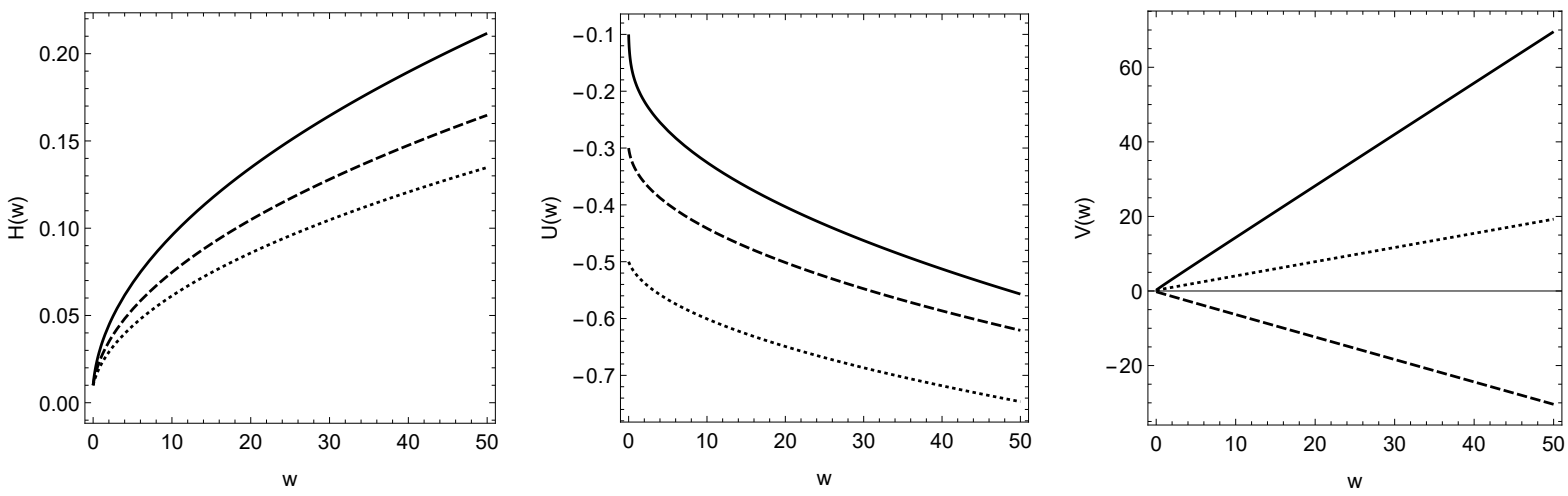

Figure 2: Qualitative evolution of $H(w), U(w)$ and $V(w)$ as they are given from the differential equation (??) for $\Omega=1$ and for different values for the initial conditions are they are presented in figures.

\subsection{Reduction with $\left\{Y_{4}, Y_{5}\right\}$}

Reduction with the Lie point symmetries $\left\{Y_{4}, Y_{5}\right\}$ provides the following system of ordinary differential equations

$$
\begin{aligned}
\frac{H_{w}}{H} & =\frac{\Omega H^{2}+w-U}{L(w)}, \\
U_{w} & =\frac{H(2 U H \Omega+w \Omega-g)}{L(w)}, \\
V_{w} & =V(U-w)^{-1},
\end{aligned}
$$

where

$$
h(t, x, y)=H(w), u(t, x, y)=U(w), v(t, x, y)=\frac{y}{t}+V(w) \text { and } w=\frac{x}{t}
$$

and

$$
L(w)=w H \Omega(H+1)+\Omega U H^{2}-g H+(w-U)^{2} .
$$

However, that solution is not accepted because it leads to an infinite value of $V(w)$. The qualitative evolution of $U(w)$ and $V(w)$ for various initial conditions is presented in Fig. 2 


\section{Conclusions}

In this work we studied the group properties of the shallow-water equations on a linear plane with the complete Coriolis force. In particular we performed a classification of the admitted Lie point symmetries which are the generators of one-parameter point transformations which leave invariant shallow-water equations.

The coordinate system has been selected such that two terms of the Coriolis force are involved in the shallowwater equations. These terms which are the Coriolis components on the axes depend upon the position on the plane. For an arbitrary position and for the more general form the shallow-water equations admit three Lie point symmetries which are the time translation and the two space translations. For specific values of the Coriolis terms new symmetries follow.

When $\phi_{0}=0$, that is, at the equator, we found that the resulting system of hyperbolic differential equations is invariant under the action of a five-dimensional Lie algebra, while, when $\phi_{0}=\frac{\pi}{2}$, that is, at the pole, the shallow-water equations admit nine Lie point symmetries. In the latter scenario the Lie point symmetries form the same algebra as the shallow-water equations without the Coriolis term. However, the two algebras are in different representations. This case has been studied before in the literature so we focused upon the case with $\phi_{0}=0$.

For the latter case we applied the new Lie point symmetries to determine similarity transformations and to write similarity solutions for the equations by using closed-form functions. We presented reductions which describe travelling-wave and scaling solutions. When it is possible, we write the closed-form solution of the shallow-water equations. However, in the remaining cases we solve numerically the reduced system and we study the qualitative behaviour of the physical variables.

This work contributes to the subject of the group properties of hyperbolic equations with emphasis in fluid dynamics. We present for the first time new solutions of the shallow-wave equations with a complete Coriolis

term. Such an analysis shows the novelty and the power of the Lie symmetries for the study of nonlinear phenomena.

In a future work we plan to extend our analysis in the case of nonlinear topographies and with possible sources.

\section{References}

[1] N.D. Mutlubas, A. Geyer and R. Quirchmayr, Nonl. Anal. Theor. Meth. Appl. 197, 111849 (2020)

[2] S.-i. Iga and Y. Matsuda, J. Atmos. Sci. 62, 2514 (2005)

[3] J. Dong and D.F. Li, J. Comp. Appl. Math. 376, 112871 (2020)

[4] J. Jung, J.H. Hwang and A.G.L. Borthwick, KSCE J. Civil Engin. 24, 1959 (2020)

[5] A. Kurganov, Y.L. Liu and V. Zeitlin, Phys. Fluids 32, 7757 (2020)

[6] M. Zhu and Y. Wang, Zeitschrift für angewandte Mathematik und Physik 71, 96 (2020)

[7] G.F. Hewitt, Heat Transfer Engineering, 4, 67 (1983)

[8] E. Goncalves, Y. Hoarau and D. Zeidan, Shock Waves, 29, 221 (2019) 
[9] D. Zeidan, P. Bähr, P. Farber, J. Gräbel and P. Ueberholz, Computer and Fluids 181, 90 (2019)

[10] E. Goncalves and D. Zeidan, Commun. Comput. Phys. 24, 167 (2018)

[11] D. Zeidan, E. Romenski, A. Slaouti and E.F. Toro, Int. J. Num. Meth. Fluids 54, 393 (2007)

[12] E. Romenski, D. Zeidan, A. Slaouti, and E. F. Toro, Hyperbolic Conservative Model for Compressible Two-Phase Flow, Reprint NI03022-NPA, Isaac Newton Institute for Mathematical Sciences, Cambridge, U.K. (2003)

[13] J. Zhai, W. Liu and L. Yuan, Computers and Fluids 134, 90 (2016)

[14] D. Zeidan, L.T. Zhang and E. Goncalves, Int. J. Appl. Mech. 12, 2050049 (2020)

[15] P.J. Dellar and R. Salmon, Physics of Fluids 17, 106601 (2005)

[16] F.M. Mahomed and P.G.L. Leach, Quaestiones Mathematicae 12, 121 (1989)

[17] S. Moyo and P.G.L. Leach,J. Math. Anal. Appl. 252, 840 (2000)

[18] P.G.L. Leach, J. Phys. A: Math. Gen. 13, 1991 (1980)

[19] A. Paliathanasis, M. Tsamparlis and M.T. Mustafa, Int. J. Geom. Meth. Mod. Phys. 12, 1550033 (2015)

[20] A.H. Kara and F.M. Mahomed, Int. J. Theor. Phys. 34, 2267 (1995)

[21] S. Jamal, Mod. Phys. Lett. A. 35, 2050068 (2020)

[22] S. Jamal and A.G. Johnpillai, Indian J. Phys. 94, 233 (2020)

[23] S. Jamal, G. Shabbir and A.S. Mathebula, IJGMMP 15, 1850191 (2018)

[24] A.A. Chesnokov, J. Appl. Mech. Techn. Phys. 49, 737 (2008)

[25] A.A. Chesnokov, Eur. J. Appl. Math. 20, 461 (2009)

[26] A. Palithanasis, Symmetry 11, 1115 (2019)

[27] X. Xin, L. Zhang, Y. Xia and H. Liu, Appl. Math. Lett. 94, 112 (2019)

[28] S. Szatmari and A. Bihlo, Comm. Nonl. Sci. Num. Sim. 19, 530 (2014)

[29] J.-G. Liu, Z.-F. Zeng, Y. He and G.-P. Ai, Int. J. Nonl. Sci. Num. Sim. 16, 114 (2013)

[30] M. Pandey, Int. J. Nonl. Sci. Num. Sim. 16, 93 (2015)

[31] A. Paliathanasis, Zeitschrift für Naturforschung A 74, 869 (2019)

[32] P. Siriwat, C. Kaewmanee and S.V. Meleshko, Int. J. Non-lin. Mechanics, 86, 185 (2016)

[33] A. Hematulin, S.V. Meleshko and S.G. Gavrilyuk, Math. Methods Appl. Sci. 302101 (2007)

[34] P. Siriwat and S.V. Meleshko, Symmetry Integr. Geom.: Methods Appl. 4, 1 (2008)

[35] V.A. Dorodnitsym and E.I. Kaptsov, Comm. Nonl. Sci. Num. Sim. 89, 105343 (2020) 
[36] S.V. Meleshko and N.F. Samatova, Comm. Nonl. Sci. Num. Sim. 90, 105337 (2020)

[37] S.V. Meleshko, Comm. Nonl. Sci. Num. Sim. 89, 105293 (2020)

[38] A. Bihlo, N. Poltavets and R.O. Popovych, Chaos 30, 073132 (2020)

[39] A. Bihlo and R.O. Popovych, Studies in Applied Mathematics 145, 291 (2020)

[40] D. Zeidan and B. Bira, Math. Meth. Appl. Sci. 42, 4679 (2019)

[41] B. Bira, T.S. Raja and D. Zeidan, Comput. Math. Appl. 71, 46 (2016)

[42] L. V. Ovsiannikov, Group analysis of differential equations, Academic Press, New York, (1982)

[43] K. S. Chou and C. Z. Qu, Acta Applicandae Mathematicae, 83, 257 (2004).

[44] F. Galas and E. W. Richter, Phys. D 50, 297 (1991).

[45] S. V. Coggeshall and J.Meyer-ter-Vehn, J. Math. Phys. 33, 3585 (1992).

[46] F. Rezvan, E.Yasar and T. Ozer, Appl. Math. Comp. 218, 974 (2011)

[47] C. Mullaert, Journal de Mathématiques Pures et Appliquées 99, 251 (2013)

[48] P.J. Olver, Applications of Lie Groups to Differential Equations, Springer-Verlag, New York, (1993)

[49] G.W. Bluman and S. Kumei, Symmetries and Differential Equations, Springer-Verlag, New York, (1989)

[50] N.H. Ibragimov, CRC Handbook of Lie Group Analysis of Differential Equations, Volume I: Symmetries, Exact Solutions, and Conservation Laws, CRS Press LLC, Florida (2000)

[51] J. Patera, R.T. Sharp, P. Winternitz and H. Zassenhaus, J. Math. Phys. 17, 986 (1976)

[52] J. Patera and P. Winternitz, J. Math. Phys. 18, 1449 (1977) 